\section{Evolução da prevalência de anemia em crianças quilombolas, segundo dois inquéritos de base populacional em Alagoas, Brasil (2008-2018)}

\author{
Trend in the prevalence of anemia in children \\ from maroon communities according to two \\ population-based surveys in Alagoas State, Brazil \\ (2008-2018)

\section{Evolución de la prevalencia de anemia en niños quilombolas, según dos encuestas de base poblacional en Alagoas, Brasil (2008-2018)}

Laíse Gabrielly Matias de Lima Santos 1 Carla Mariana Xavier Ferreira 2 Artur Belo Azevedo 2 Samara Luiza Silva Santos 1 Samir Buainain Kassar 2 Marly Augusto Cardoso 3 Haroldo da Silva Ferreira 1

doi: 10.1590/0102-311X00122520

\section{Resumo}

Objetivou-se avaliar a evolução da prevalência de anemia em crianças quilombolas de Alagoas, Brasil. Trata-se de uma análise descritiva comparando resultados de dois inquéritos domiciliares (2008; $n=950$ e 2018; $n=426$ ), envolvendo amostra das crianças de 6 a 59 meses. A anemia foi diagnosticada com hemoglobina $<110 \mathrm{~g} / \mathrm{L}$ (HemoCue). As prevalências entre os dois inquéritos foram descritas percentualmente e pela razão de prevalência $(R P)$ e intervalo de 95\% de confiança (IC95\%), calculados por regressão de Poisson. As prevalências de anemia em 2008 e 2018 foram, respectivamente, 53\% (IC95\%: 49,8-56,1) e 38\% (IC95\%: 33,4-42,6), configurando um declinio de 28,3\% (RP= 0,72; IC95\%: 0,63-0,82). Crianças de 6 a 24 meses foram mais acometidas do que aquelas de 25 a 59 meses, tanto em 2008 (72\% vs. 44\%) como em 2018 (54,8\% vs. $28,3 \%)$. Houve redução de prevalência em ambas as faixas etárias (23,9\% e 35,7\%, respectivamente). Essa redução mais pronunciada nas crianças mais velhas fez que as mais jovens passassem a ter quase o dobro da prevalência vista nas de maior idade ( $R P=1,94$; IC95\%: 1,53-2,46). Conclui-se que houve declínio expressivo da prevalência de anemia durante o período avaliado, persistindo, porém, como relevante problema de saúde pública, sobretudo entre as crianças de 6 a 24 meses. As crianças avaliadas sobrevivem em grande vulnerabilidade social, evidenciando-se que, para promover a saúde dessa população, não são suficientes ações no âmbito da saúde pública. Gestores e profissionais de saúde devem estar atentos aos dados aqui apresentados, visando à implementação de medidas para enfrentamento das iniquidades sociais e de saúde que contribuem para maior vulnerabilidade desse grupo étnico-racial.
Correspondência

H. S. Ferreira

Universidade Federal de Alagoas.

BR-104, Km 14, Maceió, AL 57072-970, Brasil.

haroldo.ufal@gmail.com

1 Universidade Federal de Alagoas, Maceió, Brasil.

2 Curso de Medicina, Centro Universitário Tiradentes, Maceió, Brasil.

3 Faculdade de Saúde Pública, Universidade de São Paulo, São Paulo, Brasil. 


\section{Introdução}

A anemia é caracterizada pela redução da quantidade de hemoglobina no sangue, com prejuízos ao transporte de oxigênio e consequente hipóxia tissular 1. Acomete cerca de um terço da população mundial ${ }^{1}$, sobretudo gestantes e crianças ${ }^{2}$. Entre estas, a prevalência no Brasil, em 2006, foi de 20,9\% 3 .

Crianças anêmicas podem apresentar prejuízos em seu crescimento e desenvolvimento, diminuição da capacidade cognitiva, redução da competência imunológica e aumento da morbimortalidade 4. Por esses motivos, a anemia é reconhecida como importante problema de saúde pública mundial 5 .

De etiologia multifatorial 6, a anemia decorrente de déficit de ferro metabolicamente disponível, conhecida como anemia ferropriva, é a forma mais frequente no Brasil e nos demais países de baixa e média renda 5. Embora de susceptibilidade universal, acomete mais frequentemente indivíduos dos estratos populacionais de menor nível socioeconômico 4,7,8.

Alagoas é um dos estados mais pobres da Federação, caracterizando-se por apresentar, em comparação aos demais, os piores indicadores sociais 9 . Compatível com esse perfil, elevada prevalência de anemia foi recentemente observada entre as crianças alagoanas $(27,4 \%) 8$. Todavia estudo realizado também em Alagoas, em 2008, encontrou valor ainda maior (52,7\%) 10 entre crianças da população quilombola desse estado.

A população quilombola encontra-se em grande vulnerabilidade social e discriminação institucional, com marcantes desigualdades quando comparada à população geral e, mais ainda, se comparada à população branca do país, razão pela qual são mais vulneráveis a uma série de agravos, inclusive à anemia $7,11,12$.

Considerando a maior vulnerabilidade da população quilombola, seria esperado que esta recebesse maior investimento por parte do poder público no sentido de priorizar a implementação de programas de promoção da saúde. Para tanto, há necessidade de informação atualizada quanto à distribuição e evolução dos agravos à saúde. Contudo, em decorrência da transição epidemiológica pela qual vem passando o país, é possível que as informações produzidas em 2008 estejam desatualizadas, sendo importante verificar qual a situação atual e como evoluiu a prevalência de anemia. Tais informações são imprescindíveis para a avaliação, implementação e reorientação de políticas públicas específicas e articuladas com as reais necessidades desse grupo étnico-racial.

Este estudo teve por objetivo avaliar a evolução da prevalência de anemia e dos níveis de hemoglobina, segundo faixa etária e sexo, em crianças quilombolas de 6 a 59 meses, conforme dois inquéritos (2008-2018) de base populacional conduzidos no Estado de Alagoas.

\section{Métodos}

Trata-se de um estudo baseado na comparação dos resultados obtidos em dois inquéritos domiciliares, ambos de delineamento transversal, realizados com dez anos de intervalo (2008 e 2018), integrantes de um projeto mais amplo, denominado Diagnóstico de Nutrição e Saúde da População das Comunidades Remanescentes dos Quilombos do Estado de Alagoas (primeira e segunda edições), doravante designados como Inquéritos I e II, respectivamente. Até 2016, Alagoas contava com 68 comunidades certificadas como quilombolas, das quais nenhuma era titulada (atualmente apenas uma comunidade possui o título de suas terras).

\section{Plano amostral}

Inquérito I: em 2008, existiam 39 comunidades quilombolas em Alagoas, mas não havia estimativa segura quanto ao número de habitantes nessas localidades. Diante disso, optou-se por estudar o universo de crianças de 6 a 59 meses, identificadas nos domicílios das 39 comunidades.

Inquérito II: em 2018, mais 29 comunidades foram reconhecidas, totalizando 68 comunidades quilombolas. O projeto maior do qual este estudo faz parte tinha por objetivo verificar a prevalência de insegurança alimentar entre as famílias quilombolas 13. Para obter uma amostra representativa dessas famílias, utilizou o processo de amostragem por conglomerados, sorteando-se 50\% das 68 comunidades. 
Para permitir maior comparabilidade com o Inquérito I, foram elegíveis todas as crianças de 6 a 59 meses identificadas nos domicílios das comunidades sorteadas. O cálculo amostral realizado a posteriori com auxílio do módulo statcalc do Epi Info, versão 7.2.1.0 (https://www.cdc.gov/epiinfo/ index.html), considerou uma prevalência de anemia de 53\% (verificada no Inquérito I), uma população de 852 crianças (amostra do Inquérito II multiplicada por 2) e um intervalo de $95 \%$ de confiança (IC95\%). Com tais parâmetros, o erro amostral considerando o número de indivíduos examinados no Inquérito II $(\mathrm{n}=426)$ foi de $3,3 \%$.

\section{Coleta de dados}

Nos dois inquéritos, os dados foram coletados por estudantes de graduação ou pós-graduação de cursos da área da saúde, treinados e supervisionados por docentes integrantes da equipe técnica, utilizando formulários estruturados previamente testados em estudos-pilotos. Estes foram realizados em comunidade rural em 2008 e, em 2018, numa das comunidades quilombolas não sorteadas para compor a amostra. No estudo-piloto, o treinamento realizado era colocado em prática e eventuais erros nos procedimentos eram discutidos e corrigidos.

A coleta dos dados ocorreu por meio de visitas domiciliares, as quais eram agendadas previamente com os líderes das comunidades. As informações sobre a criança foram prestadas pelas mães ou responsáveis. No Inquérito I, a coleta de dados foi concluída em novembro de 2008 e, no Inquérito II, em março de 2018 10,13,14.

Para a caracterização das crianças, foram obtidos dados demográficos, socioeconômicos e antropométricos: sexo, idade em meses (6-12, 13-24, 25-36, 37-48 e 49-59), cor da pele (pretos/pardos; brancos), número de residentes no domicílio $(\leq 4 ;>4)$; família usuária do Programa Bolsa Família (sim; não), classe econômica, peso e estatura.

O Programa Bolsa Família é desenvolvido pelo Governo Federal e sua principal ação é o complemento da renda das famílias, visando à redução da pobreza no Brasil (https://www.gov.br/cidadania/ pt-br/acoes-e-programas/bolsa-familia).

A classe econômica foi definida segundo Critério de Classificação Econômica Brasil 15, que distingue as famílias em ordem decrescente (A, B1, B2, C1, C2 e D/E), conforme o escore obtido a partir de um sistema de pontuação baseado no grau de instrução do chefe da família, acesso a serviços públicos (domicílio com água encanada, banheiro e localizado em rua pavimentada) e existência na residência de empregados domésticos e determinados bens de consumo (automóveis, microcomputador, máquina de lavar louça, geladeira, freezer, máquina de lavar roupa, DVD, micro-ondas, motocicleta e secadora de roupa). Quanto maior a pontuação, maior o nível econômico. Em razão da ausência de famílias pertencentes aos estratos superiores (classes A e B1), a variável foi analisada de forma dicotômica: B+C e D/E.

Para aferição da estatura, as crianças maiores de 24 meses foram medidas em posição ortostática em um estadiômetro vertical, enquanto o comprimento das crianças menores de 24 meses foi verificado na posição de decúbito dorsal em infantômetro. Ambos os equipamentos eram dotados de escala métrica inextensível com sensibilidade de $0,1 \mathrm{~cm}$. O estado nutricional das crianças foi classificado com base em escores z críticos aplicados a diferentes índices antropométricos, sendo estabelecidas as seguintes condições: baixo peso (peso-para-idade $<-2 z$ ), déficit estatural (estatura-para-idade $<-2 z$ ); magreza (índice de massa corporal-para-idade $<-2 \mathrm{z}$ ); excesso de peso (índice de massa corporal-paraidade > 2). Os dados antropométricos foram processados utilizando-se o software Anthro (https:// www.who.int/childgrowth/en/).

Foram diagnosticadas como anêmicas as crianças com concentração de hemoglobina $<110,0 \mathrm{~g} / \mathrm{L}$. Para classificar a gravidade da anemia, foram considerados os seguintes pontos de corte: leve (100,0109,9g/L); moderada $(70,0-99,9 \mathrm{~g} / \mathrm{L})$ e severa $(<70,0 \mathrm{~g} / \mathrm{L}) 6$. A hemoglobina foi medida em hemoglobinômetros portáteis (HemoCue Hb 201+ em 2008 e HemoCue Hb 301 em 2018; Ängelholm, Suécia) em gota de sangue obtida por punção da polpa digital. Segundo Rappaport et al. 16, não existem diferenças significantes para os resultados obtidos entre os modelos desse equipamento.

Para definir a importância para a saúde pública da anemia em populações a partir de sua prevalência, estabelecida por meio dos níveis sanguíneos de hemoglobina, utilizou-se a classificação proposta pela Organização Mundial da Saúde (OMS): grave ( $\geq 40 \%$ ), moderada (20\% a 39,9\%), leve (5\% a 19,9\%) e normal $(\leq 4,9 \%)$. 


\section{Critério de exclusão}

A presença da anemia falciforme foi estabelecida como critério de exclusão. No Inquérito I, essa condição foi prevista para ser identificada por meio de pergunta formulada nos seguintes termos: " $<$ nome da criança> possui algum problema de saúde?”. Esperava-se que a mãe relatasse a presença da anemia falciforme (ou de qualquer outra condição). Como nenhum caso foi identificado, houve necessidade de reformular a abordagem no Inquérito II, estabelecendo-se a pergunta de forma mais objetiva: “<nome da criança > possui anemia falciforme?". Adicionalmente, o agente de saúde era consultado sobre a existência de crianças com esse tipo de enfermidade no âmbito da comunidade. De um total de 431 crianças elegíveis para o estudo, cinco foram identificadas como portadoras dessa alteração genética.

\section{Análise estatística}

Os dados foram digitados em dupla entrada, em formulário gerado no Epi Info, versão 3.5.4. Após comparação e correção de erros de digitação, os dados foram submetidos à análise estatística. Constatada a aderência à distribuição normal (teste de Kolmogorov-Smirnov), utilizou-se o teste $t$ de Student, para comparar as concentrações médias de hemoglobina observadas em 2008 e 2018, ou ANOVA com teste de Bonferroni quando comparações múltiplas. O relacionamento entre idade e hemoglobina foi analisado com o teste de correlação de Pearson.

Para comparar as frequências segundo as variáveis sexo e faixa etária, foi utilizado o teste quiquadrado. Como medida de associação, utilizou-se a razão de prevalência (RP) e os seus respectivos IC95\%, calculados por regressão de Poisson com ajuste robusto da variância.

A tendência temporal (2008-2018) observada entre os estudos foi descrita como variação percentual [(prevalência atual-prevalência anterior)/prevalência anterior] x 100 e, também, pela RP entre os dois inquéritos. A RP foi também utilizada para descrever a associação entre prevalência de anemia com o sexo e a faixa etária da criança.

Todas as análises foram procedidas no software Stata, versão 12.0 (https://www.stata.com) e, em todas as situações, a significância estatística foi assumida quando $\mathrm{p}<0,05$.

\section{Aspectos éticos}

Os projetos relativos ao Inquérito I (no 23065.022355/2008-66) e ao Inquérito II (no 33527214.9.0000.5013) foram aprovados pelo Comitê de Ética em Pesquisa da Universidade Federal de Alagoas.

\section{Resultados}

Foram investigadas 950 crianças em 2008 e 426 em 2018. A maioria pertencia às mais baixas (D/E) classes econômicas (99\% em 2008 e em 95,1\% em 2018) e era usuária do Programa Bolsa Família (95\% em 2008 e 83,8\% em 2018). As crianças tinham, predominantemente, a cor da pele preta ou parda (82,5\% em 2008 e 90,3\% em 2018). Entre as condições antropométricas de maior prevalência, destacaram-se o déficit estatural (14,2\% em 2008 e 7,6\% em 2018), seguido pelo excesso de peso corporal (8,3\% em 2008 e 9,6\% em 2018). Essas e outras características demográficas, socioeconômicas e antropométricas das amostras se encontram apresentadas na Tabela 1.

A prevalência de anemia em 2008 foi de 53\% (IC95\%: 49,7-56,1), enquanto em 2018 foi de 38,0\% (IC95\%: 33,4-42,6), representando um declínio de 28,3\% ( $\mathrm{RP}=$ 0,72; IC95\%: 0,63-0,82). Esse declínio foi comum a todas as faixas etárias (Tabela 2). Apesar disso, nas faixas etárias mais jovens, a magnitude permaneceu em patamares superiores a 40\%, mantendo-se na condição de grave problema de saúde pública. Em 2018, a prevalência de anemia entre as crianças de 6 a 24 meses foi cerca de duas vezes maior em relação àquela observada para as crianças de 25 a 59 meses $(54,8 \%$ vs. $28,3 \%$. RP $=1,94$; IC95\%: 1,53-2,46), conforme consta na Tabela 2. 


\section{Tabela 1}

Distribuição de crianças ( 6 a 59 meses) das comunidades remanescentes de quilombos de Alagoas, segundo variáveis socioeconômicas, demográficas e antropométricas, conforme dois estudos transversais (2018-2018). Alagoas, Brasil.

\begin{tabular}{|c|c|c|c|c|}
\hline Variável/Categorias & $\begin{array}{l}2008 \\
\text { n (\%) }\end{array}$ & $\begin{array}{l}2018 \\
\text { n (\%) }\end{array}$ & Valor de $p$ * & $\begin{array}{c}\text { Variação } \\
(\%) * *\end{array}$ \\
\hline Sexo & & & 0,596 & \\
\hline Masculino & $483(50,8)$ & $210(49,3)$ & & $-3,0$ \\
\hline Feminino & $467(49,2)$ & $216(50,7)$ & & 3,0 \\
\hline Faixa etária (meses) & & & 0,398 & \\
\hline $6-12$ & $102(10,7)$ & $51(12,0)$ & & 12,1 \\
\hline $13-24$ & $202(21,3)$ & $106(24,9)$ & & 16,9 \\
\hline $25-36$ & $219(23,1)$ & $99(23,2)$ & & 0,4 \\
\hline $37-48$ & $230(24,2)$ & $88(20,7)$ & & $-14,5$ \\
\hline $49-59$ & $197(20,7)$ & $82(19,3)$ & & $-6,8$ \\
\hline Cor da pele & & & $<0,001$ & \\
\hline Preta/Parda & $593(82,5)$ & $382(90,3)$ & & 9,5 \\
\hline Branca & $126(17,5)$ & $41(9,7)$ & & $-44,6$ \\
\hline Baixo peso (peso-para-idade $<-2 z$ ) & & & 0,237 & \\
\hline Sim & $42(4,4)$ & $13(3,1)$ & & $-29,5$ \\
\hline Não & $904(95,6)$ & $409(96,9)$ & & 1,4 \\
\hline Déficit estatural (altura-para-idade $<-2 z$ ) & & & 0,001 & \\
\hline Sim & $132(14,2)$ & $32(7,6)$ & & $-46,5$ \\
\hline Não & $797(85,8)$ & $390(92,4)$ & & 7,7 \\
\hline Magreza (índice de massa corporal-para-idade $<-2 z$ ) & & & 0,155 & \\
\hline Sim & $22(2,4)$ & $5(1,2)$ & & $-50,0$ \\
\hline Não & $908(97,6)$ & $414(98,8)$ & & 1,2 \\
\hline Excesso de peso (índice de massa corporal-para-idade $<2 z$ ) & & & 0,444 & \\
\hline Sim & $77(8,3)$ & $40(9,6)$ & & 15,7 \\
\hline Não & $853(91,7)$ & $379(90,4)$ & & $-1,4$ \\
\hline Número de residentes no domicílio & & & $<0,001$ & \\
\hline$>4$ & $533(56,3)$ & $171(40,1)$ & & $-28,8$ \\
\hline$\leq 4$ & $414(43,7)$ & $255(59,9)$ & & 37,1 \\
\hline Família usuária do Programa Bolsa Família & & & $<0,001$ & \\
\hline Sim & $689(95,0)$ & $357(83,8)$ & & $-11,8$ \\
\hline Não & $36(5,0)$ & $69(16,2)$ & & 224,0 \\
\hline Classe econômica da família *** & & & $<0,001$ & \\
\hline $\mathrm{D} / \mathrm{E}$ & $940(99,0)$ & $405(95,1)$ & & $-3,9$ \\
\hline$B+C$ & $10(1,0)$ & $21(4,9)$ & & 390,0 \\
\hline
\end{tabular}

* Valor de $\mathrm{p}$ segundo o teste qui-quadrado;

** Variação percentual $(\Delta \%)=[(2018-2008) / 2008]$ x 100;

*** Classificação econômica da Associação Brasileira de Empresas de Pesquisa 15.

Em 2008, meninos e meninas apresentaram prevalências semelhantes ( $\mathrm{p}=$ 0,315): 51,3\% vs. 54,6\%, respectivamente. Contudo houve maior prevalência entre o sexo masculino em 2018: 43,8\% vs. 32,4\% $(\mathrm{p}=0,015)$.

Na Figura 1, encontram-se ilustradas as prevalências de anemia segundo suas diferentes categorias. Evidencia-se o declínio em todos os níveis, embora, conforme os IC95\%, não significante para a anemia leve. Todavia houve declínio significante para a anemia moderada e o completo desaparecimento da forma grave em 2018. 
Tabela 2

Prevalência, variação percentual e razão de prevalência (RP) de anemia por faixa etária, segundo dois inquéritos (2008-2018) em crianças de 6 a 59 meses de comunidades remanescentes dos quilombos no Estado de Alagoas, Brasil.

\begin{tabular}{|c|c|c|c|c|c|c|c|c|}
\hline \multirow{2}{*}{$\begin{array}{l}\text { Faixa etária } \\
\text { (meses) }\end{array}$} & \multicolumn{2}{|c|}{ Amostra } & \multicolumn{2}{|c|}{ Anemia } & \multirow{2}{*}{$\begin{array}{c}\text { Variação } \\
(\%) *\end{array}$} & \multirow{2}{*}{$\begin{array}{c}2008 \text { vs. } 2018 \\
\text { RP (IC95\%) } \\
\text { [valor de p] }\end{array}$} & \multicolumn{2}{|c|}{ Conforme faixa etária } \\
\hline & $\begin{array}{c}2008 \\
n\end{array}$ & $\begin{array}{c}2018 \\
n\end{array}$ & $\begin{array}{c}2008 \\
\% \text { (IC95\%) }\end{array}$ & $\begin{array}{c}2018 \\
\%(\text { IC95\%) }\end{array}$ & & & $\begin{array}{c}2008 \\
\text { RP (IC95\%) } \\
\text { [valor de p] }\end{array}$ & $\begin{array}{c}2018 \\
\text { RP (IC95\%) } \\
\text { [valor de p] }\end{array}$ \\
\hline $06-12$ & 102 & 51 & $\begin{array}{c}76,5 \\
(68,2 ; 84,8)\end{array}$ & $\begin{array}{c}68,6 \\
(55,7 ; 81,5)\end{array}$ & $-10,3$ & $\begin{array}{c}0,89(0,72 ; 1,11) \\
{[0,324]}\end{array}$ & $\begin{array}{c}2,12(1,71 ; 2,63) \\
{[<0,001]}\end{array}$ & $\begin{array}{c}2,56(1,71 ; 3,83) \\
{[<0,001]}\end{array}$ \\
\hline $13-24$ & 202 & 106 & $\begin{array}{c}70,2 \\
(63,4 ; 76,2)\end{array}$ & $\begin{array}{c}48,1 \\
(38,5 ; 57,7) * *\end{array}$ & $-31,1$ & $\begin{array}{c}0,69(0,55 ; 0,86) \\
{[0,001]}\end{array}$ & $\begin{array}{c}1,95(1,58 ; 2,39) \\
{[<0,001]}\end{array}$ & $\begin{array}{c}1,79(1,19 ; 2,70) \\
{[0,005]}\end{array}$ \\
\hline $25-36$ & 219 & 99 & $\begin{array}{c}52,3 \\
(45,9 ; 59,1)\end{array}$ & $\begin{array}{c}35,4 \\
(25,9 ; 44,8) * *\end{array}$ & $-32,6$ & $\begin{array}{c}0,67(0,50 ; 0,90) \\
{[0,009]}\end{array}$ & $\begin{array}{c}1,45(1,16 ; 1,82) \\
{[0,001]}\end{array}$ & $\begin{array}{c}1,32(0,84 ; 2,06) \\
{[0,226]}\end{array}$ \\
\hline $37-48$ & 230 & 88 & $\begin{array}{c}42,6 \\
(36,2 ; 49,0)\end{array}$ & $\begin{array}{c}21,6 \\
(12,9 ; 30,3) * *\end{array}$ & $-49,3$ & $\begin{array}{c}0,51(0,33 ; 0,78) \\
{[0,002]}\end{array}$ & $\begin{array}{c}1,18(0,93 ; 1,50) \\
{[0,170]}\end{array}$ & $\begin{array}{c}0,80(0,47 ; 1,38) \\
{[0,427]}\end{array}$ \\
\hline $49-59$ & 197 & 82 & $\begin{array}{c}36,0 \\
(29,3 ; 42,8)\end{array}$ & $\begin{array}{c}26,8 \\
(17,2 ; 36,5)\end{array}$ & $-25,3$ & $\begin{array}{c}0,74(0,50 ; 1,11) \\
{[0,152]}\end{array}$ & 1,00 & 1,00 \\
\hline $06-24$ & 304 & 157 & $\begin{array}{c}72,0 \\
(67,0 ; 77,1)\end{array}$ & $\begin{array}{c}54,8 \\
(46,9 ; 62,6)\end{array}$ & $-23,9$ & $\begin{array}{c}0,76(0,65 ; 0,89) \\
{[0,001]}\end{array}$ & $\begin{array}{c}1,64(1,47 ; 1,83) \\
{[0,001]}\end{array}$ & $\begin{array}{c}1,94(1,53 ; 2,46) \\
{[0,001]}\end{array}$ \\
\hline $25-59$ & 646 & 269 & $\begin{array}{c}44,0 \\
(40,1 ; 47,8)\end{array}$ & $\begin{array}{c}28,3 \\
(22,8 ; 33,6)\end{array}$ & $-35,7$ & $\begin{array}{c}0,64(0,52 ; 0,79) \\
{[<0,001]}\end{array}$ & 1,00 & 1,00 \\
\hline Total & 950 & 426 & $\begin{array}{c}53,0 \\
(49,8 ; 56,1)\end{array}$ & $\begin{array}{c}38,0 \\
(33,4 ; 42,6)\end{array}$ & $-28,3$ & $\begin{array}{c}0,72(0,63 ; 0,82) \\
{[<0,001]}\end{array}$ & - & - \\
\hline
\end{tabular}

IC95\%: intervalo de $95 \%$ de confiança.

* $\Delta \%=[(2018-2008) / 2008] \times 100$;

* Diferença estatisticamente significante de acordo com o IC95\%.

Figura 1

Distribuição de crianças de 6 a 59 meses, segundo diferentes categorias de anemia: resultados de dois inquéritos (20082018) realizados nas comunidades remanescentes dos quilombos do Estado de Alagoas, Brasil.

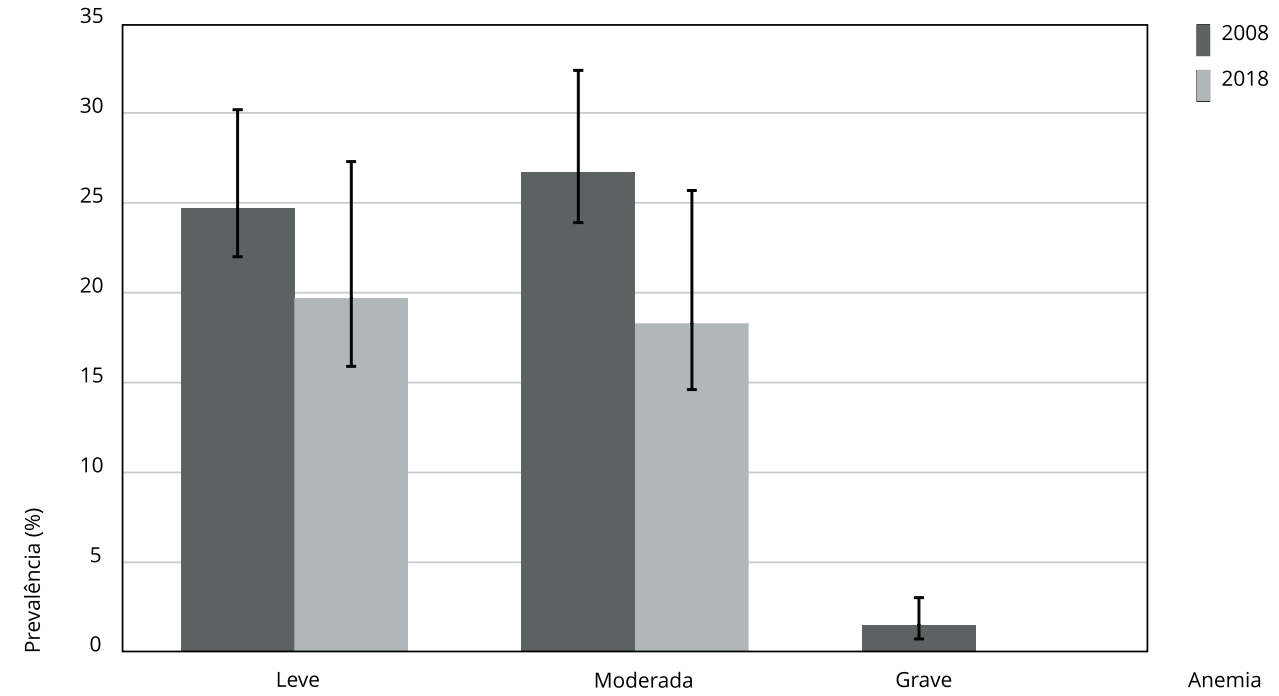


Em coerência com essas reduções, houve aumento dos valores médios de hemoglobina, independentemente de sexo e idade (Tabela 3). Em todas as situações, houve diferença significante quando comparados os valores em 2008 em relação a 2018, exceção feita para a faixa etária de 6-12 meses e de 49-59 meses. Nos dois inquéritos houve correlação positiva entre idade e concentração de hemoglobina: $\mathrm{r}=0,31 \mathrm{em} 2008$ e $\mathrm{r}=0,32 \mathrm{em} 2018$. Na análise segundo o sexo, em cada momento avaliado (2008 ou 2018), não houve diferença estatisticamente significante para os valores médios de hemoglobina.

Tabela 3

Médias e desvios-padrão de hemoglobina por faixa etária ou sexo em dois inquéritos (2008-2018) em crianças de 6 a 59 meses de comunidades remanescentes dos quilombos no Estado de Alagoas, Brasil.

\begin{tabular}{|c|c|c|c|c|c|}
\hline \multirow[t]{3}{*}{ Variáveis } & \multicolumn{2}{|c|}{ Amostra } & \multicolumn{2}{|c|}{ Hemoglobina (g/L) } & \multirow[t]{3}{*}{ Valor de $p$ * } \\
\hline & 2008 & 2018 & 2008 & 2018 & \\
\hline & $(n=950)$ & $(n=426)$ & & & \\
\hline \multicolumn{6}{|c|}{ Faixa etária (meses) } \\
\hline $6-12$ & 102 & 51 & $99,7 \pm 15,8 a$ & $104,2 \pm 12,5 e$ & 0,076 \\
\hline $13-24$ & 202 & 106 & $102,0 \pm 16,4 a$ & $108,0 \pm 14,3 e, g$ & $0,001 * *$ \\
\hline $25-36$ & 219 & 99 & $107,8 \pm 15,5 b$ & $112,4 \pm 14,7 f, g, h$ & $0,014 * *$ \\
\hline $37-48$ & 230 & 88 & $111,6 \pm 15,0 c$ & $118,2 \pm 11,7 f, i$ & $<0,001 \star \star$ \\
\hline $49-59$ & 197 & 82 & $113,7 \pm 13,8^{d}$ & $116,2 \pm 14,9 f, h, i$ & 0,182 \\
\hline \multicolumn{6}{|l|}{ Sexo } \\
\hline Masculino & 483 & 210 & $108,2 \pm 16,3 a$ & $111,3 \pm 14,8 b$ & $0,020 * *$ \\
\hline Feminino & 467 & 216 & $107,4 \pm 15,7 a$ & $113,1 \pm 14,2 b$ & $<0,001 \star \star *$ \\
\hline \multirow[t]{2}{*}{ Total } & 950 & 426 & $107,8 \pm 16,0$ & $112,2 \pm 14,5$ & $<0,001 * *$ \\
\hline & & & $(106,8 ; 108,9)$ & $(110,8 ; 113,6)$ & \\
\hline
\end{tabular}

* Valor de p segundo o teste t de Student;

** Diferença estatisticamente significante.

Nota: quando, na mesma coluna, letras distintas indicam diferença estatisticamente significante $(p<0,05)$; letras iguais indicam ausência dessa condição $(p \geq 0,05)$. Cálculos realizados por análise de variância com teste de Bonferroni.

\section{Discussão}

Os dados ora apresentados evidenciam a grande vulnerabilidade social em que se encontra a população quilombola investigada, constatando-se que mais de $95 \%$ das famílias pertencem às mais baixas classes econômicas $(\mathrm{D} / \mathrm{E})$, frequência muito superior à distribuição encontrada para a população brasileira (28,3\%) 15. Estudo realizado com a população de Alagoas em 201513 encontrou que $51,2 \%$ das famílias pertenciam às classes D/E. Esse valor, apesar de elevado, considerando a população brasileira como referência, ainda é muito inferior ao encontrado na população quilombola ora avaliada.

Nesse contexto, ressalta-se a dependência que essa população tem em relação às políticas públicas de modo geral e, em particular, dos programas de transferência de renda. Em 2008, quase todas as crianças pertenciam a famílias usuárias do Programa Bolsa Família, fato que, provavelmente, contribuiu para a relevante redução na prevalência de anemia, mesmo tendo havido diminuição da cobertura do programa (de $95 \%$ em 2008 para 83,8\% em 2018). Uma possível explicação para esse fato seria que a menor cobertura do Programa Bolsa Família no segundo inquérito teria ocorrido devido ao desmonte das políticas sociais que vem sendo empreendido no país a partir de 2016 13,17. No entanto, pelo curto espaço de tempo decorrido, a redução da cobertura do Programa Bolsa Família não foi 
capaz de reverter o processo de melhoria da condição nutricional que estava em curso. Contudo só a partir de um novo inquérito será possível ter maior clareza quanto aos efeitos das atuais prioridades governamentais.

Como já referido, houve importante redução da prevalência de anemia no segundo inquérito em relação ao primeiro. Todavia os valores observados em 2018 indicam que a anemia é um agravo de grande magnitude entre as crianças quilombolas e, apesar da razoável redução observada, continua a representar um relevante problema de saúde pública nessa população.

Em termos percentuais, houve redução da prevalência de anemia da ordem de 28,3\% entre os dois inquéritos. Estudo semelhante realizado com crianças da população de Alagoas também encontrou redução em relação aos valores do inquérito inicial: a anemia passou de 45,1\% em 2005 para 27,4\% em 2015 8, caracterizando redução de 39,1\% e, portanto, mais expressiva que aquela observada para as crianças quilombolas.

A prevalência de 38\% observada em 2018 nas crianças deste estudo caracteriza a situação como problema moderado, porém a dois pontos percentuais de alcançar o ponto de corte para ser considerado como grave problema de saúde pública 6.

São escassas as publicações, nos últimos dez anos, com foco na prevalência de anemia em crianças quilombolas brasileiras, o que limita a comparação dos nossos achados.

Quanto à etiologia, embora existam diversos outros fatores que levam à anemia, a deficiência de ferro está frequentemente envolvida, sobretudo na vigência de altas prevalências e em países de média ou baixa renda 18 . Assim, a prevenção da anemia passa necessariamente pela correção desse problema. Por esse motivo, em 2005 foi implantado no Brasil o Programa Nacional de Suplementação de Ferro (PNSF), o qual, além da suplementação e outras ações, preconiza também a educação alimentar e nutricional 15. Dessa forma, seria esperado encontrar prevalência de anemia em patamar mais próximo da aceitável ( $\leq 5 \%$ ), conforme preconiza a OMS, o que ficou distante de ser alcançado.

Em estudo anterior sobre a efetividade do PNSF em Alagoas 19, verificou-se que baixa cobertura, baixa adesão e deficiências nas ações educativas explicavam parte da baixa efetividade do programa nesse estado 20. Mesmo com esses óbices, seria razoável esperar que as prevalências de anemia observadas nas crianças quilombolas estivessem num patamar semelhante ao verificado nas crianças alagoanas em geral. Porém estas foram muito menos afetadas que as crianças aqui estudadas: $27,4 \%$ em 2015 vs. 38\% em 2018. Esse diferencial pode estar relacionado às iniquidades sociais e à maior exposição às carências nutricionais, e, também, devido à discriminação institucional 21.

Nesse aspecto, Silva et al. 22 relataram que as comunidades quilombolas são historicamente desfavorecidas e submetidas a processo de invisibilidade e desigualdades quanto ao reconhecimento de seus direitos. Acrescentam que "as politicas de igualdade adotadas a partir de 2003 representaram um grande avanço no enfrentamento às iniquidades existentes nessas comunidades. Contudo, apesar dos avanços conquistados, em 2016 o Brasil ainda era um dos países mais desiguais do mundo, e as diferenças em relação a cor/raça resultam em sequelas expressivas para o país" 22 (p. 3).

Nesse cenário de dificuldades sociais e econômicas 23 , é possível supor que as crianças aqui investigadas não tiveram acesso a uma alimentação adequada e saudável; consequentemente, estariam mais expostas não apenas à ingestão insuficiente de ferro e de outros nutrientes relacionados à anemia, mas aos demais micro e macronutrientes necessários a um bom estado nutricional. Esses aspectos estão condizentes com a alta prevalência de anemia encontrada e, também, com o perfil antropométrico das crianças investigadas. Entre os problemas nutricionais identificados por antropometria, a condição mais prevalente foi o déficit estatural, que, em nível populacional, é um indicador de desnutrição crônica 24 , a qual acometeu 14,2\% das crianças quilombolas alagoanas em 2008. Assim como a anemia, também se observou declínio de sua prevalência, passando para 7,6\% em 2018.

Esses achados mais uma vez revelam as disparidades existentes entre as crianças quilombolas e as crianças não quilombolas de Alagoas. Ferreira et al. 25 conduziram inquéritos de base populacional nesse estado e encontraram, em anos próximos aos dos inquéritos realizados neste estudo, prevalência de desnutrição crônica sempre em níveis mais elevados entre os quilombolas, respectivamente: 11,2\% em 2005 vs. 14,2\% em 2008, e 3,2\% em 2015 vs. 7,6\% em 2018. No caso do déficit estatural, os autores 25 atribuíram a expressiva redução observada entre os dois inquéritos, sobretudo, às políticas públicas implementadas durante o período. Porém, mais uma vez, fica evidente que fatores promo- 
tores de saúde foram mais efetivos na população não quilombola. Enquanto a redução na prevalência de desnutrição crônica entre crianças da população geral do estado foi de $71,4 \%$, esse percentual para as crianças quilombolas foi de $46,5 \%$.

Ao contrário da tendência de declínio observada para as carências nutricionais (anemia e desnutrição), os valores relativos ao excesso de peso nas crianças quilombolas apresentaram evolução positiva, semelhantemente ao também observado para as crianças alagoanas não quilombolas 25 , porém em menor magnitude: 8,3\% em 2008 e 9,6\% em 2018 vs. 7,5\% em 2005 e 14,9\% em 2015, respectivamente.

Esse crescimento da magnitude do excesso de peso nesse contexto pode ser atribuído a duas possíveis causas: à programação metabólica induzida pela desnutrição no início da vida 26 e/ou ao padrão alimentar baseado em alimentos de alta densidade energética e de baixo valor nutritivo, a exemplo dos alimentos ultraprocessados 27 . Em cenários caracterizados por processos históricos de iniquidades sociais e insegurança alimentar, os processos de ajuste metabólico em crianças com déficit estatural resultante da desnutrição crônica, se por um lado garantem sua sobrevivência, por outro levam o organismo a apresentar menor taxa metabólica (menor gasto de energia), maior suscetibilidade aos efeitos de dietas ricas em gordura, menor habilidade para oxidação lipídica e danos fisiológicos relacionados ao controle do apetite. Esses mecanismos aumentam a suscetibilidade ao excesso de peso 28,29 . O baixo poder aquisitivo diminui o acesso à alimentação adequada e se associa a maior exposição ao consumo de alimentos ultraprocessados ${ }^{30}$. Nessas situações também prevalece a baixa escolaridade, a qual contribui para opções alimentares não saudáveis (como substituir frutas regionais por refrigerantes ou sucos artificiais) ${ }^{14}$. Esse padrão alimentar, aliado à baixa taxa de metabolismo basal, induzido pela programação metabólica, pode levar à obesidade e suas comorbidades, configurando-se em mais um agravo característico das populações em vulnerabilidade social. Assim, no contexto em que prevalece a anemia como problema de saúde pública, outras questões relevantes para a saúde dessas populações também merecem atenção.

Embora a análise dos fatores associados à anemia não faça parte dos objetivos aqui propostos, em virtude dos inúmeros relatos existentes na literatura quanto à relação dessa enfermidade com a menor faixa etária da criança 31,32,33,34, julgou-se pertinente investigar como foi o relacionamento dessas variáveis neste estudo, constatando-se que, quanto menor a idade da criança, maior a ocorrência da anemia.

Essa abordagem fica ainda mais evidente quando são analisados os valores sanguíneos de hemoglobina das crianças conforme as cinco faixas etárias investigadas, verificando-se um claro efeito dose-resposta. Em 2008, essas concentrações evoluíram da menor faixa etária até a maior, da seguinte forma (g/L): 99,7, 102,0, 107,8, 111,6 e 113,7. Comparando cada valor com aquele imediatamente subjacente, apenas entre os dois primeiros (relativos às faixas etárias de 6-12 e de 13 -24 meses) não houve significância estatística. Embora em 2018 tenha havido incrementos nesses valores em todas as faixas etárias, manteve-se a mesma tendência de correlação positiva entre idade e nível de hemoglobina. No inquérito de 2018, os níveis médios (g/L) dessa hemoproteína foram 104,2, 108,0, 112,4, 118,2 e 116,2, respectivamente.

A maior suscetibilidade à anemia em crianças com idade inferior a 24 meses pode ser atribuída à maior velocidade de crescimento que ocorre nesse período da vida, o que se faz acompanhar de elevação nos requerimentos de ferro. A isso se acrescenta o fato de que, até os seis meses de vida, os estoques adquiridos na fase fetal são exauridos. Assim, a adequada introdução da alimentação complementar tem papel preponderante na prevenção de carências nutricionais, especialmente do ferro 33,35 .

Durante os dois primeiros anos de vida podem ocorrer algumas situações que aumentam as possibilidades de desenvolvimento de anemia: introdução precoce de alimentos; interrupção do aleitamento materno exclusivo antes dos seis meses; inadequação da alimentação complementar ou daquela oferecida após o desmame; e maior exposição a doenças parasitárias e infecciosas nessa fase de maior vulnerabilidade biológica 33,36.

Tal como Pessoa et al. 37 ao avaliarem 556 crianças de 6 a 24 meses do Município de Vespasiano (Minas Gerais), neste estudo, ser do sexo masculino se associou à maior prevalência de anemia, embora somente no Inquérito II. Isso pode ser atribuído não apenas à maior velocidade de crescimento característico do sexo masculino, levando a uma maior necessidade de ferro, o qual pode não ser totalmente suprido pela dieta, mas também devido a fatores genéticos e metabólicos, conforme 
sugerido por Domellöf et al. 38 ao investigarem 263 crianças (121 suecas e 142 hondurenhas) aleatoriamente alocadas aos quatro meses de idade para receber suplementação de ferro ou placebo até os nove meses de idade.

Em nosso estudo, embora as diferenças de prevalência segundo o sexo respaldem essas observações, quando a análise tem por base os níveis de hemoglobina, essa relação resta fragilizada, pois as diferenças entre meninos e meninas não foram suficientemente grandes a ponto de se descartar que tenham ocorrido por conta do acaso (g/L): 108,2 vs. 107,4 em 2008 (p=0,429) e 111,3 vs. 113,1 ( $\mathrm{p}=$ $0,190)$, respectivamente.

O padrão alimentar das crianças não foi analisado, o que constitui uma limitação deste estudo, pois impossibilita reconhecer a importância dessa variável na etiologia da anemia. Outra limitação foi que o diagnóstico da anemia considerou apenas a quantificação da hemoglobina, não sendo possível identificar os distintos tipos de anemia conforme sua etiologia. Contudo, como já referido, é amplamente reconhecido que em cenários onde a anemia na infância constitui problema de saúde pública, a anemia nutricional, sobretudo aquela por deficiência de ferro, é a forma predominante 20,39,40.

Além dessas limitações, é importante considerar que os inquéritos utilizados para embasar este trabalho não foram desenhados para atender aos objetivos aqui propostos. Assim, alguns óbices metodológicos tiveram origem nesse fato, a exemplo de planos amostrais diferentes e do não planejamento de critérios de exclusão específicos, tais como doenças crônicas ou infecciosas, que podem interferir nos valores de hemoglobina. Contudo o estudo apresenta adequada abordagem analítica, as amostras obtidas foram representativas do universo de interesse e, nos dois inquéritos, os dados foram coletados por entrevistadores bem treinados e supervisionados, utilizando métodos preconizados internacionalmente. Por tais motivos, o estudo tem validade interna e responde aos objetivos propostos.

Este estudo constitui uma importante fonte de informação a ser utilizada, principalmente por gestores responsáveis por promover ações de enfrentamento da problemática apresentada, de modo a reduzir as prevalências vigentes para patamares aceitáveis, evitando-se a persistência da anemia como problema de saúde pública.

De forma mais abrangente, considerando a grande vulnerabilidade social a que a população estudada se encontra submetida 21,23 e o fato de que as condições socioeconômicas, psicológicas, étnico-raciais e culturais são aspectos que influenciam de modo relevante os processos que determinam o padrão de saúde e a distribuição de doenças em indivíduos e coletividades, denominados determinantes sociais em saúde 41, é imprescindível que ações estruturantes sejam garantidas a essas populações, tais como acesso à educação, formação profissional e geração de emprego e renda, além de acesso à infraestrutura de serviços públicos, alimentação adequada, saneamento básico e saúde, bem como a outros direitos garantidos na Constituição Federal.

Essa recomendação está atrelada à determinação política para eliminar as iniquidades existentes quando se comparam os povos quilombolas (e outras minorias étnicas, como os povos indígenas) com a população geral do país. Nesse aspecto, é importante destacar as fases descritas por Dalosto et al. 21 , a partir de uma análise histórica das políticas públicas destinadas às comunidades quilombolas brasileiras.

Segundo esses autores 21 , é possível caracterizar quatro fases que vão desde o período colonial até os dias atuais: (1) ocorreu durante o período em que existia a escravidão no Brasil e foi marcada pela perseguição e repressão contra os quilombos e seus habitantes; (2) iniciada ao final do período escravocrata brasileiro, caracterizou-se pela inexistência de políticas públicas destinadas aos quilombolas; (3) teve início "com a discussão e a positivação na Constituição Federal do direito de tombamento dos sítios históricos dos quilombos, proteção da cultura específica das comunidades quilombolas e de titulação dos territórios das comunidades, e os momentos posteriores até o governo do ex-presidente Luís Inácio Lula da Silva" 21 (p. 164); e (4) se distingue pela criação de mecanismos jurídicos institucionais destinados a garantir a aplicabilidade dos direitos previstos na legislação e o estabelecimento de programas e políticas públicas destinados à população quilombola. Esta última ainda se encontra em curso, pois, apesar dos retrocessos ocorridos nos governos de Michel Temer e de Jair Messias Bolsonaro, ainda não houve um completo desmonte das instituições, dos instrumentos normativos e das políticas destinadas às comunidades quilombolas. No entanto, com base no posicionamento do atual presidente, conjectura-se a grande possibilidade de maiores retrocessos. Com o desmonte na estrutura especializada e a entrada na agenda formal do Governo Federal de diversas pautas que não favorecem 
os povos em maior vulnerabilidade social, é plausível afirmar que as ações do governo destinadas à superação da miséria e à inclusão sofrerão mais prejuízos, o que irá piorar as condições de vida das comunidades quilombolas brasileiras e acarretará um possível retrocesso nos diversos avanços conseguidos nos últimos anos.

Dados citados em artigo de Melo \& Silva 23, com base em diversos estudos, revelam que existe mais de um milhão de quilombolas no Brasil, a maioria vivendo em zonas rurais, em condição de extrema pobreza, sob precárias condições sanitárias, dependentes de assistência do governo (sobretudo do Programa Bolsa Família) e com baixa escolaridade. Esse perfil reflete muito o que foi demonstrado neste estudo. Embora nossos resultados não possam ser extrapolados para outros contextos, sendo limitados à população quilombola do Estado de Alagoas, tal similaridade indica que, muito provavelmente, podem representar a realidade da maioria das comunidades quilombolas dispersas por todo o território nacional.

\section{Conclusão}

No período compreendido entre 2008 e 2018, verificou-se um importante declínio na prevalência de anemia, todavia mantendo-se em alto patamar, a apenas dois pontos percentuais de permanecer na condição anterior de grave problema de saúde pública (condição observada nos dois inquéritos para as crianças de 6 a 24 meses).

É importante ressaltar a destacada vulnerabilidade social das famílias às quais pertencem as crianças avaliadas. Fundamenta essa afirmação o fato de que mais de $90 \%$ delas pertencem às mais baixas classes econômicas (D/E) e que mais de $80 \%$ dependem do Programa Bolsa Família para sobreviver. Diante disso, fica evidente que, para a promoção da saúde da população investigada, são necessárias não apenas ações específicas no âmbito da saúde pública.

Profissionais e gestores das políticas públicas devem considerar essas características para a implementação de medidas de prevenção e controle, enfatizando a necessidade de políticas redutoras das iniquidades sociais que tornam os povos quilombolas especialmente suscetíveis a uma série de problemas de saúde e à má qualidade de vida.

\section{Colaboradores}

L. G. M. L. Santos, C. M. X. Ferreira, A. B. Azevedo, S. L. S. Santos e S. B. Kassar contribuíram na preparação dos dados, análise estatística, interpretação dos resultados e redação da versão preliminar do manuscrito. M. A. Cardoso colaborou na análise estatística, interpretação dos resultados e revisão geral do estudo. H. S. Ferreira realizou a análise estatística e a revisão final do texto. Todos os autores aprovaram a versão final do texto a ser publicado

\section{Informações adicionais}

ORCID: Laíse Gabrielly Matias de Lima Santos (0000-0003-2887-1943); Carla Mariana Xavier Ferreira (0000-0001-7814-9436); Artur Belo Azevedo (0000-0003-3783-006X); Samara Luiza Silva Santos (0000-0002-0974-9160); Samir Buainain Kassar (0000-0003-1068-6360); Marly Augusto Cardoso (0000-0003-0973-3908); Haroldo da Silva Ferreira (0000-0002-1789-3138).

\section{Agradecimentos}

Ao Conselho Nacional de Desenvolvimento Científico e Tecnológico (CNPq) e à Fundação de Amparo à Pesquisa do Estado de Alagoas (FAPEAL) pelo financiamento. 


\section{Referências}

1. World Health Organization. Nutritional anaemias: tools for effective prevention and control. Geneva: World Health Organization; 2017.

2. McCarthy EK, Chaoimh CN, Kenny LC, Hourihane JO, Irvine AD, Murray DM, et al. Iron status, body size, and growth in the first 2 years of life. Matern Child Nutr 2018; 14:e12458.

3. Mujica-Coopman MF, Brito A, López de Romaña D, Ríos-Castillo I, Coris H, Olivares M. Prevalence of anemia in Latin America and the Caribbean. Food Nutr Bull 2015; 36(2 Suppl):S119-28.

4. World Health Organization. Iron deficiency anemia: assessment, prevention, and control. A guide for programme managers. Geneva: World Health Organization; 2001.

5. World Health Organization. The global prevalence of anaemia in 2011. Geneva: World Health Organization; 2015.

6. World Health Organization. Haemoglobin concentrations for the diagnosis of anaemia and assessment of severity. Geneva: World Health Organization; 2011.

7. Matos CCSA, Tourinho FSV. Saúde da população negra: como nascem, vivem e morrem os indivíduos pretos e pardos em Florianópolis (SC). Rev Bras Med Fam Comunidade 2018; 13:1-13.

8. Vieira RC, Livramento AR, Calheiros MS, Ferreira CMX, Santos TR, Assunção ML, et al. Prevalence and temporal trend (2005-2015) of anaemia among children in Northeast Brazil. Public Health Nutr 2018; 21:868-76.

9. Neri MC, Vaz FM, Souza PF. Duas décadas de desigualdade e pobreza no Brasil medidas pela PNAD/IBGE. Brasília: Instituto de Pesquisa Econômica Aplicada; 2013.

10. Ferreira HS, Lamenha MLD, Xavier Júnior AFS, Cavalcante JC, Santos AM. Nutrição e saúde das crianças das comunidades remanescentes dos quilombos no Estado de Alagoas, Brasil. Rev Panam Salud Pública 2011; 30:51-8.

11. Souza Filho CFM, Prioste F. Quilombos no Brasil e direitos socioambientais na América Latina. Revista Direito e Práxis 2017; 8:2903-26.

12. Silva EF, Pontes DR, Milano GB. Terras quilombolas no Brasil: das técnicas de dominação colonial ao reconhecimento democráticoconstitucional. Revista Brasileira de Sociologia do Direito 2017; 4:126-47.

13. Costa NS, Santos MO, Carvalho CPO, Assunção ML, Ferreira HS. Prevalence and factors associated with food insecurity in the context of the economic crisis in Brazil. Curr Dev Nutr 2017; 1:e000869.

14. Leite FM, Ferreira HS, Bezerra MK, Assunção ML, Horta BL. Consumo alimentar e estado nutricional de pré-escolares das comunidades remanescentes dos quilombos do estado de Alagoas. Rev Paul Pediatr 2013; 31:444-51.
15. Associação Brasileira de Empresas de Pesquisa. Critério de Classificação Econômica Brasil. Alterações na aplicação do Critério Brasil, válidas a partir de 01/Jun/2019. São Paulo: Associação Brasileira de Empresas de Pesquisa; 2019.

16. Rappaport AI, Karakochuk CD, Whitfield KC, Kheang KM, Green TJ. A method comparison study between two hemoglobinometer models (Hemocue Hb 301 and $\mathrm{Hb} 201+$ ) to measure hemoglobin concentrations and estimate anemia prevalence among women in Preah Vihear, Cambodia. Int J Lab Hematol 2017; 39:95-100.

17. Silva IC. Reflexões sobre o debate da "nova" classe média no Brasil. PRACS: Revista Eletrônica de Humanidades do Curso de Ciências Sociais da Unifap 2020; 12:75-89.

18. Centro Brasileiro de Análise e Planejamento. Pesquisa Nacional de Demografia e Saúde da Criança e da Mulher: PNDS, 2006. Brasília: Ministério da Saúde/São Paulo: Centro Brasileiro de Análise e Planejamento; 2009.

19. Calheiros MSC. Efetividade do Programa Nacional de Suplementação de Ferro: estudo de base populacional com lactentes de 6 a 18 meses do Estado de Alagoas [Dissertação de Mestrado]. Maceió: Universidade Federal de Alagoas; 2017.

20. World Health Organization. Who guideline: use of multiple micronutrient powders for point-of-use fortification of foods consumed by infants and young children aged 6-23 months and children aged 2-12 years. Geneva: World Health Organization; 2016.

21. Dalosto CD, Dalosto JA, Oliveira CL. As políticas públicas para as comunidades quilombolas no Brasil. In: Lacerda GB, organizador. As políticas públicas frente a transformação da sociedade. Ponta Grossa: Atena Editora; 2019. p. 155-66.

22. Silva BMA, Silveira VNC, Padilha LL, Frota MTBA. Situação de insegurança alimentar e nutricional em famílias quilombolas maranhenses. Demetra (Rio J.) 2020; 15:43636.

23. Melo MF, Silva HP. Doenças crônicas e os determinantes sociais da saúde em comunidades quilombolas do Pará, Amazônia, Brasil. Rev ABPN 2015; 7:168-89.

24. Ferreira HS. Anthropometric assessment of children's nutritional status: a new approach based on an adaptation of Waterlow's classification. BMC Pediatrics 2020; 20:65.

25. Ferreira HS, Albuquerque GT, Santos TR, Barbosa RL, Cavalcante AL, Duarte LE, et al. Stunting and overweight among children in Northeast Brazil: prevalence, trends (19922005-2015) and associated risk factors from repeated cross-sectional surveys. BMC Public Health 2020; 20:736. 
26. Martins VJ, Florêncio TMT, Grillo LP, Carmo PFM, Martins PA, Clemente AP, et al. Longlasting effects of undernutrition. Int J Environ Res Public Health 2011; 8:1817-46.

27. Filgueiras AR, Almeida VBP, Nogueira PCK, Domene SMA, Silva CE, Sesso R, et al. Exploring the consumption of ultra-processed foods and its association with food addiction in overweight children. Appetite 2019; 135:137-45.

28. Ferreira HS, Xavier Júnior AF, Assunção ML, Uchôa TC, Lira-Neto AB, Nakano RP. Developmental origins of health and disease: a new approach for the identification of adults who suffered undernutrition in early life. Diabetes Metab Syndr Obes 2018; 11:543-51.

29. Ferreira HS, Luna AA, Florêncio TM, Assunção ML, Horta BL. Short stature is associated with overweight but not with high energy intake in low-income Quilombola women. Food Nutr Bull 2017; 38:216-25.

30. Soares DA, Barreto SM. Indicadores nutricionais combinados e fatores associados em população Quilombola no Sudoeste da Bahia, Brasil. Ciênc Saúde Colet 2015; 20:821-32.

31. Assunção MCF, Santos IS, Barros AJD, Gigante DP, Victora CG. Anemia em menores de seis anos: estudo de base populacional em Pelotas, RS. Rev Saúde Pública 2007; 41:328-35.

32. Oliveira CS, Cardoso MA, Araújo TS, Muniz PT. Anemia em crianças de 6 a 59 meses e fatores associados no Município de Jordão, Estado do Acre, Brasil. Cad Saúde Pública 2011; 27:1008-20.

33. Rocha DS, Capanema FD, Pereira Netto M, Franceschini SCC, Lamounier JA. Prevalence and risk factors of anemia in children attending daycare centers in Belo Horizonte-MG. Rev Bras Epidemiol 2012; 15:675-84.

34. Varela R, Russo S, Ferreira F, Lequini N, Savio E, Gonzalez M, et al. Prevalencia de anemia en niñas/os de 6 a 48 meses que concurren a dos CAIF de la Ciudad de Salto. Rev Salud Pública 2019; 23:69-77.
35. Vieira AC, Diniz A, Cabral P, Oliveira R, Lóla $\mathrm{M}$, Silva $\mathrm{S}$, et al. Avaliação do estado nutricional de ferro e anemia em crianças menores de 5 anos de creches públicas. J Pediatr (Rio J.) 2007; 83:370-6.

36. Domellöf M, Braegger C, Campoy C, Colomb V, Decsi T, Fewtrell M, et al. Iron requirements of infants and toddlers. J Pediatr Gastroenterol Nutr 2014; 58:119-29.

37. Pessoa MC, Jansen AK, Velásquez-Meléndez G, Lopes JD, Beinner MA. Anemia em crianças e fatores associados em região urbana. REME Rev Min Enferm 2011; 15:54-61.

38. Domellöf M, Lönnerdal B, Dewey KG, Cohen RJ, Rivera LL, Hernell O. Sex differences in iron status during infancy. Pediatrics 2002; 110:545-52.

39. Rodrigues VC, Mendes BD, Gozzi A, Sandrini F, Santana RG, Matioli G. Deficiência de ferro, prevalência de anemia e fatores associados em crianças de creches públicas do oeste do Paraná, Brasil. Rev Nutr 2011; 24:407-20.

40. Gondim SSR, Diniz AS, Souto RA, Bezerra RGS, Albuquerque EC, Paiva AA. Magnitude, time trends and factors associate with anemia in children in the state of Paraíba, Brazil. Rev Saúde Pública 2012; 46:649-56.

41. Buss PM, Pellegrini Filho A. A saúde e seus determinantes sociais. Physis (Rio J.) 2007; 17:77-93. 


\section{Abstract}

The study aimed to assess trends in the prevalence of anemia in children from quilombos (maroon communities) in Alagoas State, Brazil. This was a descriptive study comparing the results of two household surveys (2008; $n=950$ and 2018; $n=$ 426), involving a sample of children from 6 to 59 months of age. Anemia was diagnosed as hemoglobin $<110 \mathrm{~g} / \mathrm{L}$ (HemoCue). Prevalence rates between the two surveys were described by percentage and by prevalence ratio (PR) and 95\% confidence interval (95\%CI), calculated by Poisson regression. Prevalence rates for anemia in 2008 and 2018 were $53 \%$ (95\% CI: 49.8-56.1) and 38\% (95\% CI: 33.4-42.6), respectively, or a decrease of 28.3\% ( $R P=0.72$; 95\%CI: 0.63-0.82). Children 6 to 24 months of age had higher anemia rates than those 25 to 59 months of age, both in 2008 (72\% vs. $44 \%$ ) and in 2018 (54.8\% vs. 28.3\%). There was a reduction in prevalence in both age brackets $(23.9 \%$ and $35.7 \%$, respectively). This sharper decline in older children meant that younger children had nearly double the prevalence rate compared to older children ( $P R=1.94$; 95\%CI: $1.53-$ 2.46). In conclusion, there was a major decline in prevalence of anemia during the period studied, but anemia persisted as a relevant public health problem, especially in children 6 to 24 months of age. The children in the sample are exposed to harsh social vulnerabilities, evidencing that health promotion for this population requires more than actions in the public health sphere itself. Healthcare workers and administrators should be alert to the data presented here, aimed at implementation of measures to confront the social and health iniquities that contribute to greater vulnerability in this ethnic-racial group.

Iron-deficiency Anemia; Health of Ethnic Minorities; Child Health; Health Surveys

\section{Resumen}

El objetivo fue evaluar la evolución de la prevalencia de anemia en niños quilombolas de Alagoas, Brasil. Se trata de un análisis descriptivo, comparando resultados de dos encuestas domiciliarias (2008; $n=950$ y 2018; $n=426)$, implicando en la muestra a niños de 6 a 59 meses. La anemia se diagnosticó con hemoglobina $<110 \mathrm{~g} / \mathrm{L}$ (HemoCue). Las prevalencias entre los dos cuestionarios fueron descritas porcentualmente y por la razón de prevalencia (RP) e intervalo de $95 \%$ de confianza (IC95\%), calculados por regresión de Poisson. Las prevalencias de anemia en 2008 y 2018 fueron, respectivamente, 53\% (IC95\%: 49, 8-56,1) y 38\% (IC95\%: 33,4-42,6), configurando un declive de un 28,3\% ( $R P=0,72$; IC95\%: 0,63-0,82). Los niños de 6 a 24 meses estuvieron más afectados que aquellos de 25 a 59 meses, tanto en 2008 (72\% vs. 44\%), como en 2018 (54,8\% vs. 28,3\%). Hubo una reducción de prevalencia en ambas franjas etarias (23,9\% y 35,7\%, respectivamente). Esta reducción más pronunciada en niños mayores provocó que los más jóvenes pasasen a tener casi el doble de la prevalencia, vista en aquellos de mayor edad $(R P=$ 1,94; IC95\%: 1,53-2,46). Se concluye que hubo un declive expresivo de la prevalencia de anemia durante el periodo evaluado, persistiendo, no obstante, como un relevante problema de salud pública, sobre todo entre los niños de 6 a 24 meses. Los niños evaluados sobreviven bajo una gran vulnerabilidad social, evidenciándose que para la promoción de la salud de esa población no son suficientes acciones en el ámbito de la salud pública. Gestores $y$ profesionales de salud deben estar atentos a los datos aquí presentados, con el fin de implementar medidas para enfrentar las inequidades sociales $y$ de salud que contribuyen a una mayor vulnerabilidad de ese grupo étnico-racial.

Anemia Ferropénica; Salud de las Minorías Étnicas; Salud del Niño; Encuestas Epidemiológicas
Recebido em 13/Mai/2020

Versão final reapresentada em 07/Dez/2020

Aprovado em 04/Jan/2021 